\title{
Incidence of discontinuation of angiotensin-converting enzyme inhibitors due to cough, in a primary healthcare centre in Singapore
}

Lai Peng $\mathrm{Ng}^{1}$, MMed, FCFP, Paul Soo Chye Goh ${ }^{1}$, MMed, FCFP

INTRODUCTION The incidence of cough induced by angiotensin-converting enzyme (ACE) inhibitors has been reported to be $5 \%-20 \%$, with less than half of affected patients requiring discontinuation due to persistent cough. However, the incidence in the local Asian population has not been studied. This study aimed to objectively evaluate the incidence of discontinuation of ACE inhibitors due to cough, in a primary healthcare centre in Singapore.

METHODS We retrospectively reviewed the medical records, both electronic and written, of patients who attended Tampines Polyclinic to identify those who were newly prescribed ACE inhibitors. The written medical records were analysed to identify patients who discontinued the use of ACE inhibitors and to find out the reasons for discontinuation. RESULTS A total of 424 patients were identified during the study period. Out of the 424 patients, 129 (30.4\%) discontinued the use of ACE inhibitors due to cough. Overall, 90 (21.2\%) patients who were initially started on ACE inhibitors were eventually switched to angiotensin receptor blockers (ARBs).

CONCLUSION In our cohort, the incidence of discontinuation of ACE inhibitors due to cough is higher than most other studies. The relationship between ethnicity and tolerance of medications should not be underestimated. As there is a high incidence of discontinuation of ACE inhibitors due to cough in the local population, ARBs may be a reasonable substitute as a first-line medication, if clinically indicated.

Keywords: ACE inhibitors, cough, incidence

\section{INTRODUCTION}

Angiotensin-converting enzyme (ACE) inhibitors are widely used in the treatment of hypertension, and renal and cardiovascular conditions. It is reported to be well tolerated by a high percentage of patients. However, dry cough is a common nuisance side effect with this class of medication, and the cough can be severe enough to require discontinuation of these medications. ${ }^{(1)}$

The incidence of ACE inhibitor-induced cough has been reported to be $5 \%-20 \% .^{(2)}$ A prospective study found that the incidence of cough necessitating the discontinuation of ACE inhibitors was reported to be $9.3 \%$. ${ }^{(3)}$ Few studies have suggested a relationship between ethnicity and the prevalence of cough due to ACE inhibitors. ${ }^{(4-6)}$ The prevalence of cough requiring discontinuation of $\mathrm{ACE}$ inhibitor therapy was found to be significantly higher among subjects of African ${ }^{(4)}$ and Chinese ethnicity. ${ }^{(5,6)}$ In a blinded, retrospective case control study conducted in Hong Kong, interviews were carried out among patients taking enalapril or captopril. ${ }^{(5)}$ The prevalence of persistent cough was reported to be $44 \%$, with approximately $10 \%$ of the patients withdrawing from treatment because of it. ${ }^{(5)}$

We hypothesise that the local incidence of ACE inhibitor discontinuation due to cough is higher than that reported in the Caucasian population. ${ }^{(7,8)}$ We believe that the objective data derived from our study will aid local physicians in their selection of drugs for their patients. Appropriate counselling can also be given to patients initiated on ACE inhibitors. Our study aimed to determine the incidence of discontinuation of ACE inhibitors due to cough, in a primary healthcare centre in Singapore.

\section{METHODS}

This study retrospectively reviewed the medical records of patients who attended Tampines Polyclinic, a public primary healthcare clinic in Singapore. Calculation of the needed sample size was derived using Machin et al's sample size table. ${ }^{(9)}$ Based on this table, a sample size of at least 370 patients was required for an estimated incidence of discontinuation of ACE inhibitors due to cough (in our local population) of $40 \%$, with $5 \%$ precision.

Patients prescribed ACE inhibitors for the first time at the study centre during the study period (i.e. between 1 July 2005 and 1 March 2006) were included in the study (Fig. 1). Using the clinic's electronic database, a list of patients who were prescribed the ACE inhibitors enalapril, lisinopril, captopril and perindopril was generated. These ACE inhibitors were selected as these were the only ACE inhibitors available at the study centre during the study period. The following patients were excluded from the study: (a) patients who defaulted on follow-up or were transferred to another institution within a year of initiation of ACE inhibitors; (b) patients whose ACE inhibitors were discontinued by other clinics for reasons that were not

${ }^{1}$ SingHealth Polyclinics - Tampines, Singapore

Correspondence: Dr Ng Lai Peng, Consultant, Tampines Polyclinic, 1 Tampines Street 41, Singapore 529203.ng.lai.peng@singhealth.com.sg 


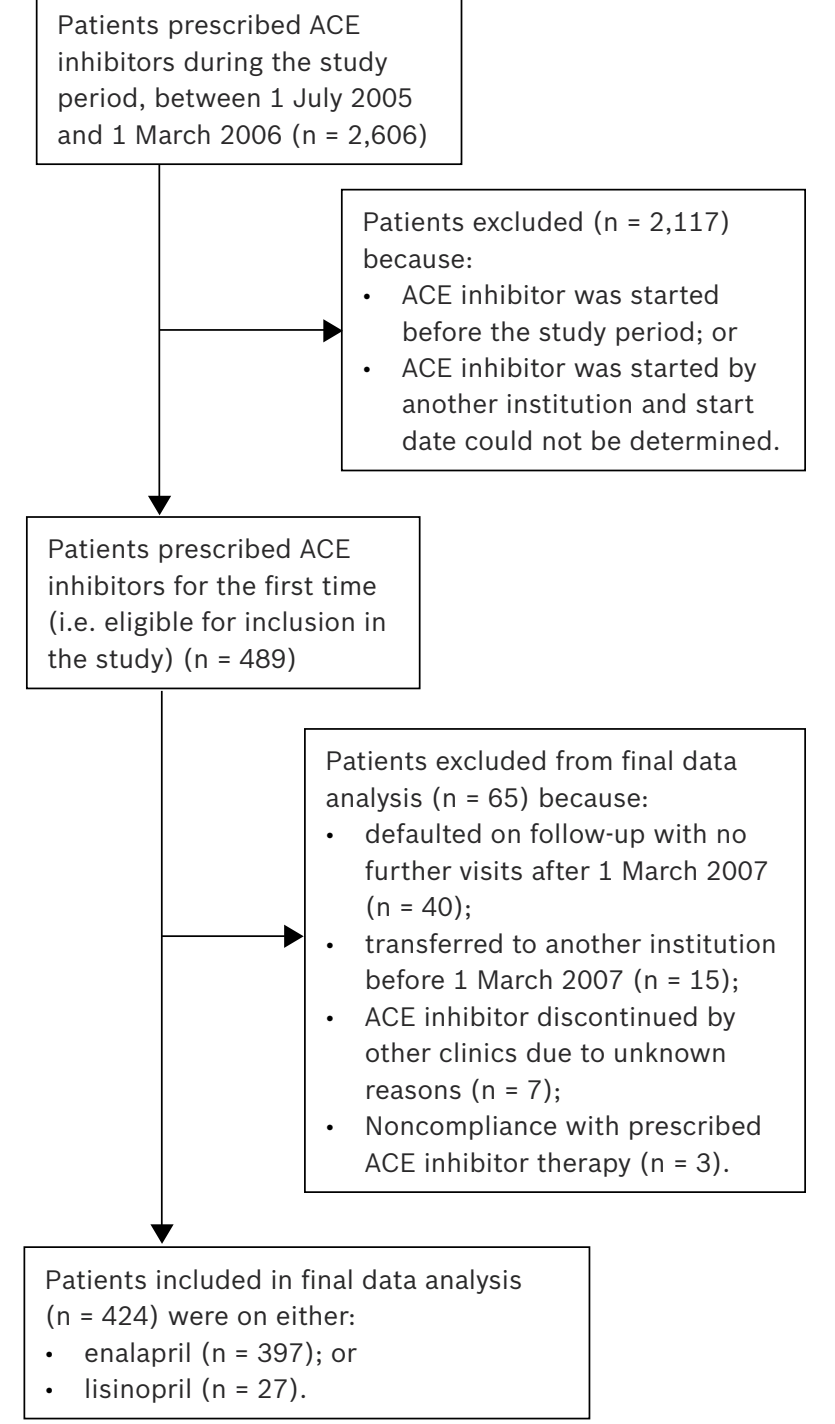

Fig. 1 Flowchart shows the selection process of patients for inclusion in the study. The medical records were reviewed between 1 August and 15 September 2007.

documented; and (c) patients who were not compliant or did not take the ACE inhibitors prescribed. Such patients were excluded as the reviewers were not able to determine whether the patient had any adverse side effect from the ACE inhibitors.

Data, including the demographic data of the patients, was retrieved and reviewed between 1 August and 15 September 2007. Two reviewers independently reviewed the individual written medical records; information on the patients' continuation or discontinuation of ACE inhibitors was gathered solely from the written entries in the medical records. In cases of uncertainty, the two reviewers reviewed the written medical records together to arrive at a consensus. For the purpose of the present study, we ensured that there was at least a year's interval between each patient's first prescription of the ACE inhibitors and the data collection period. Thus, patients included in the study would have been on intended therapy of ACE inhibitors for an adequate period of time (i.e. at least a year). Reasons for
Table I. Sociodemographic data of the study cohort $(n=424)$.

\begin{tabular}{lr}
\hline Variable & No. (\%) \\
\hline Gender & $220(51.9)$ \\
Male & $204(48.1)$ \\
Female & \\
Ethnicity & $289(68.2)$ \\
Chinese & $112(26.4)$ \\
Malay & $16(3.8)$ \\
Indian & $7(1.7)$ \\
Other & \\
Comorbidity* & $303(71.5)$ \\
Diabetes mellitus & $120(28.3)$ \\
Hypertension (without diabetes mellitus) & $5(1.2)$ \\
Congestive cardiac failure & $5(1.2)$ \\
Chronic obstructive pulmonary disease & $19(4.5)$ \\
Asthma &
\end{tabular}

the discontinuation of ACE inhibitors were obtained from the written medical record of each patient; these reasons included cough and hyperkalaemia, among others. There was more than one reason for discontinuing ACE inhibitors in some patients.

This study and a waiver of informed consent were approved by SingHealth Polyclinics Institution Review Board. Consent was not obtained from the patients, as this was a retrospective review.

\section{RESULTS}

Patients initiated on ACE inhibitors at the study centre during the study period (i.e. between 1 July 2005 and 1 March 2006) were eligible for inclusion in the study. Although 489 patients were found to be eligible for inclusion, 65 patients were excluded from data analysis, leaving only 424 patients for the final data analysis (Fig. 1). Of the 65 patients who were excluded, 40 defaulted on follow-up with no further clinic visits after 1 March 2007; it was not known whether these patients had continued or discontinued the ACE inhibitors as this could not be determined based on the medical records. Before 1 March 2007, 15 patients were either transferred to another primary care doctor upon the patient's request or referred to tertiary centres for their medical conditions. Thus, we also could not determine whether these 15 patients had continued using ACE inhibitors based on the medical records. There were also three patients who either did not take the ACE inhibitors prescribed or were not compliant with the medication - for these patients, the presence or absence of any adverse effect from the ACE inhibitors could not be determined based on their medical records.

Of the 424 patients included in the final data analysis, 397 and 27 were initiated on enalapril and lisinopril, respectively. No patient was initiated on captopril or perindopril during the study period. The demographic profile of the study population is shown in Table I. Our cohort consisted mainly of patients of Chinese ethnicity, followed by Malay ethnicity and then Indian ethnicity, similar to the ethnic composition of Singapore's 


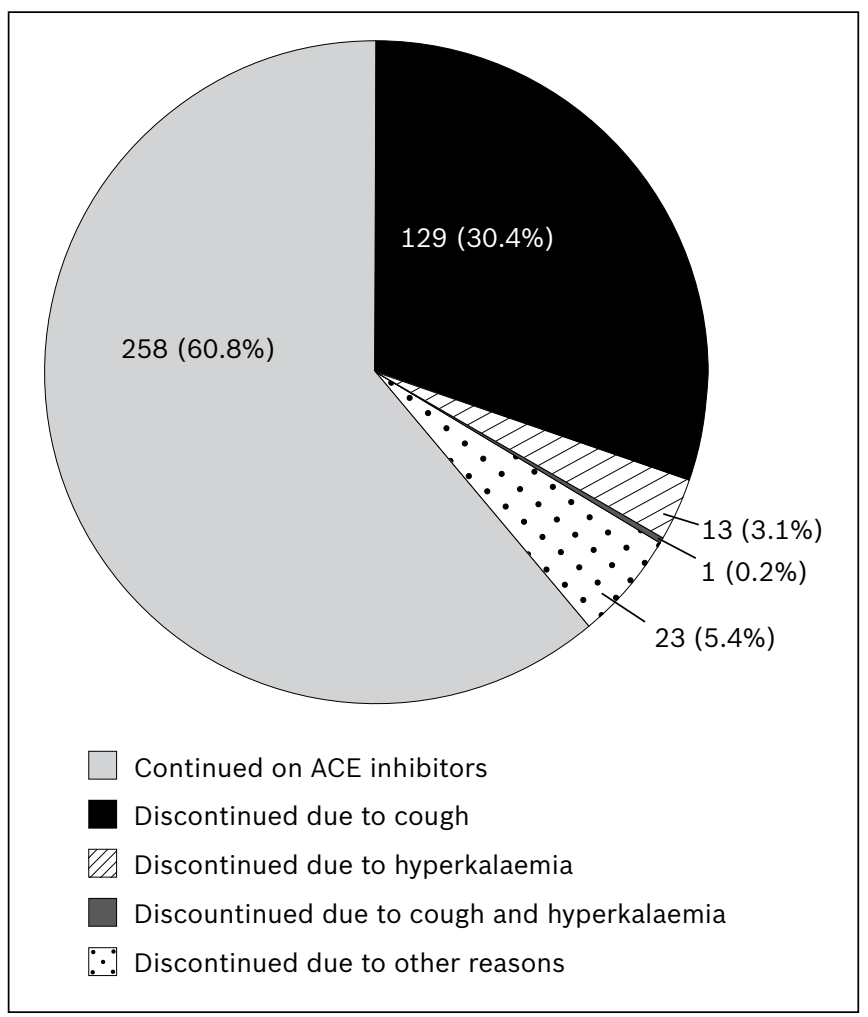

Fig. 2 Pie chart shows the incidence of angiotensin-converting enzyme (ACE) inhibitor discontinuation in our study population $(n=424)$.

Table II. Duration of angiotensin-converting enzyme inhibitor therapy before discontinuation due to cough $(n=129)$.

\begin{tabular}{ll}
\hline Duration (mths) & No. $(\%)$ \\
\hline$<1$ & $25(19.4)$ \\
$1-6$ & $66(51.2)$ \\
$6-12$ & $25(19.4)$ \\
$>12$ & $13(10.1)$ \\
\hline
\end{tabular}

population. However, our study had a higher proportion of Malay patients $(26.4 \%)$ than that of the general population in Singapore (13.4\%). ${ }^{(10)}$

Among the 424 patients, the use of ACE inhibitors was discontinued in 166 (39.2\%). In all, ACE inhibitors were discontinued due to cough in 129 (30.4\%) patients (Fig. 2). If we included the 65 patients who were excluded from the final data analysis and assume that these 65 patients had discontinued their ACE inhibitors due to cough, the incidence of discontinuation of ACE inhibitors due to cough would be $39.7 \%$ among the initial 489 patients eligible for inclusion in the study. Conversely, if these 65 patients did not discontinue the use of ACE inhibitors due to cough, the incidence of discontinuation of ACE inhibitors due to cough would be $26.4 \%$ (out of 489 patients). Among the patients who discontinued the use of ACE inhibitors due to cough ( $n=129$ ), a majority discontinued the use of ACE inhibitors within six months of therapy (Table II). Out of the 424 patients who were started on ACE inhibitors, 90 (21.2\%) were eventually switched to angiotensin receptor blockers (ARBs).
In our study, a higher proportion of female patients discontinued the use of ACE inhibitors due to cough; 72 out of the 204 (35.3\%) female patients discontinued the use of ACE inhibitors due to cough, compared to 57 out of the 220 (25.9\%) male patients $(p=0.036)$.

\section{DISCUSSION}

Our hypothesis that the incidence of discontinuation of ACE inhibitors due to cough is much higher in the local population than that reported in other studies ${ }^{(1-3,7,8)}$ was confirmed in the present study, which showed that approximately $30 \%$ of the patients discontinued ACE inhibitors due to cough. In postmarketing studies, the incidence of cough was reported to be as low as $1 \% .^{(7,8)}$ In the Physicians' Desk Reference, the reported incidence of cough associated with enalapril is $1.3 \% .{ }^{(1)}$ In a prospective clinical trial, the incidence of ACE inhibitor-induced cough is reported to be $18.6 \%$. $^{(3)} \mathrm{A}$ meta-analysis of randomised clinical trials reported the pooled weighted incidence of cough associated with enalapril to be $11.5 \%$, and the rate of withdrawal from enalapril due to cough was $2.6 \%$.(1)

Studies have suggested that there may be ethnicity-related differences with regard to the prevalence of cough attributed to ACE inhibitor therapy. ${ }^{(4-6,11)}$ An American study examining the discontinuation rates of lisinopril due to cough found that $34 \%$ of Chinese Americans experience chronic cough that was severe enough to warrant discontination their medication; this proportion is significantly higher than the $13 \%$ found in the general American population. ${ }^{(6)}$ In our study population (which consisted of $68 \%$ Chinese), the incidence of discontinuation of ACE inhibitors due to cough is similar to the aforementioned incidence observed among Chinese Americans. Our study reaffirms the possibility that ethnic differences affect the tolerability of ACE inhibitors. This suggests that Asians may have a different response to certain drugs, and the results of studies conducted in non-Asian populations may not always be applicable to Asian populations. Investigators should thus consider including Asians in future clinical trials before extrapolating results to any Asian population. In our study population, we found that $30.1 \%, 30.4 \%$ and $43.8 \%$ of the Chinese, Malay and Indian patients, respectively, discontinued their ACE inhibitors due to cough. As the primary objective of the present study was to evaluate the incidence of discontinuation of ACE inhibitors due to cough in the local population, the study sample did not include an adequate number of patients from the different ethnic groups; only $3.8 \%$ of our study population consisted of Indians (Table I). Hence, we are unable to draw any conclusion on the possible ethnic differences affecting patient reaction to ACE inhibitors. Future studies could focus on this area, so as to better understand the impact of ethnicity on ACE inhibitor-induced cough.

Ethnic differences with respect to cough reflex sensitivity has been previously postulated. ${ }^{(5)}$ Although a study conducted to evaluate the ethnic differences of cough reflex sensitivity 
among Caucasian, Indian and Chinese patients yielded no significant ethnic difference, ${ }^{(12)}$ this possibility cannot be excluded and should be evaluated in future studies. Although the present study was not designed to investigate possible gender differences in the incidence of ACE inhibitor discontinuation due to cough, our study's finding of a higher incidence in female patients, as compared to male patients, was consistent with the findings of other studies. ${ }^{(13-15)}$

A limitation of the present study is that the methodology used does not allow for the determination of any causaleffect relationships. We are unable to draw conclusions about the actual incidence of cough induced solely by ACE inhibitors because the decision to discontinue ACE inhibitors (due to disabling cough) was based on the individual physician's clinical judgement, and each physician would have different thresholds and practices. However, the present study was undertaken with the aim of studying the incidence of discontinuation of ACE inhibitors in real-life clinical practice.

Although there was a total of four ACE inhibitors available from the study centre's pharmacy during the study period, our study cohort had only been prescribed enalapril or lisinopril. Due to the inconvenience posed by its dosing frequency, captopril, being an ACE inhibitor that requires thrice-daily dosing, was probably not a popular option among physicians. Due to its cost, perindopril was also not commonly initiated in the study centre during the study period. The study centre also saw patients who were initiated on captopril before the study period and patients who were initiated on perindopril from other institutions. However, these patients were not eligible for inclusion in the present study as we only included patients who were initiated on ACE inhibitors at our study centre during the study period.

A strength of the present study is that it had a relatively large sample size within a single study centre. The present study was conducted in a public primary healthcare centre in Singapore, which manages a substantial proportion of patients with chronic diseases. The study centre's pharmacy is situated within the premises of the clinic, and patients fill up their prescriptions at the onsite pharmacy. The close proximity of the pharmacy to the clinic allowed us to directly obtain data based on the actual number of patients filling up their prescriptions rather than data based solely on the number of doctors' prescriptions. Clinical data was relatively complete as the study was conducted in a single study centre where all written medical records were kept.

While our study population had representation from all of Singapore's major ethnic groups, our study had a higher proportion of Malay patients than that in the Singapore population. This should be taken into consideration if the present study's results are to be generalised to all patients in Singapore. ACE inhibitors are a useful class of drugs used in the management of hypertension, and renal and cardiovascular diseases. However, the side effect of cough can be disabling. Our study provides local physicians with an objective figure on the local incidence of discontinuation of ACE inhibitors due to cough. This will aid them in their choice of a first-line therapy for patients with indications for ACE inhibitors. For patients who develop disabling cough, ARB is commonly prescribed in place of ACE inhibitors. Patients prescribed ACE inhibitors can be informed of the local incidence of discontinuation due to cough.

In conclusion, the present study found a $30 \%$ incidence in the discontinuation of ACE inhibitors due to cough, in a primary healthcare centre in Singapore. This incidence is much higher than that reported in previous studies. ${ }^{(1-3,7,8)}$ Local physicians may consider using ARBs as a first-line medication, if clinically indicated.

\section{ACKNOWLEDGEMENTS}

The authors are grateful to Dr Sim Kok Ping, Ms Sharon Fan (for assisting in the acquisition of data), the staff of Tampines Polyclinic, and the Department of Education and Research, Singhealth Polyclinics, Singapore, for their support.

\section{REFERENCES}

1. Bangalore S, Kumar S, Messerli FH. Angiotensin-converting enzyme inhibitor associated cough: deceptive information from the Physicians' Desk Reference. Am J Med 2010; 123:1016-30.

2. Israili ZH, Hall WD. Cough and angioneurotic edema associated with angiotensin-converting enzyme inhibitor therapy. A review of the literature and pathophysiology. Ann Intern Med 1992; 117:234-42.

3. Ravid D, Lishner M, Lang R, Ravid M. Angiotensin-converting enzyme inhibitors and cough: a prospective evaluation in hypertension and in congestive heart failure. J Clin Pharmacol 1994; 34:1116-20.

4. Elliott WJ. Higher incidence of discontinuation of angiotensin converting enzyme inhibitors due to cough in black subjects. Clin Pharmacol Ther 1996; 60:582-8.

5. Woo KS, Nicholls MG. High prevalence of persistent cough with angiotensin converting enzyme inhibitors in Chinese. Br J Clin Pharmacol 1995; 40:141-4.

6. Tseng DS, Kwong J, Rezvani F, Coates AO. Angiotensin-converting enzymerelated cough among Chinese-Americans. Am J Med 2010; 123:183.e11-5.

7. Chalmers D, Whitehead A, Lawson DH. Postmarketing surveillance of captopril for hypertension. Br J Clin Pharmacol 1992; 34:215-23.

8. Cooper WD, Sheldon D, Brown D, et al. Post-marketing surveillance of enalapril: experience in 11,710 hypertensive patients in general practice. J R Coll Gen Pract 1987; 37:346-9.

9. Machin D, Campbell MJ, Fayers $P$, et al. Sample size tables for clinical studies. 2nd ed. Oxford: Blackwell Science, 1997.

10. Census of Population 2010 Advance Census Release. Department of Statistics, Ministry of Trade and Industry, Republic of Singapore [online]. Available at www.singstat.gov.sg/. Accessed January 28, 2014.

11. Nishizawa A. Angiotensin-converting enzyme inhibitor induced cough among Asians. Proceedings of UCLA Healthcare 2000; 4:35-38. Available at: www.med.ucla.edu/modules/wfsection/article.php?articleid=135 Accessed January 28, 2014.

12. Dicpinigaitis PV, Allusson VR, Baldanti A, Nalamati JR. Ethnic and gender differences in cough reflex sensitivity. Respiration 2001; 68:480-2.

13. Dicpinigaitis PV. Angiotensin-converting enzyme inhibitor-induced cough: ACCP evidence-based clinical practice guidelines. Chest 2006; 129(1 Suppl):169S-173S.

14. Yeo WW, Ramsay LE. Persistent dry cough with enalapril: incidence depends on method used. J Hum Hypertens 1990; 4:517-20.

15. Yesil S, Yesil M, Bayata S, Postaci N. ACE inhibitors and cough. Angiology 1994; 45:805-8. 